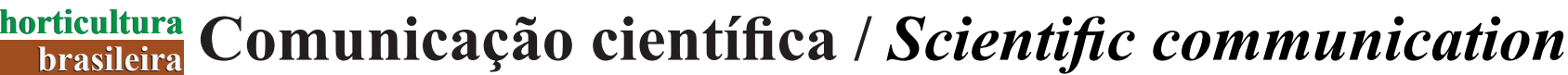

RESENDE GM; COSTA ND. 2014. Dose econômica de nitrogênio na produtividade e armazenamento de cultivares de cebola. Horticultura Brasileira 32: 357-362. DOI - http://dx.doi.org/10.1590/S0102-05362014000300019

\section{Dose econômica de nitrogênio na produtividade e armazenamento de cultivares de cebola}

\author{
Geraldo M Resende; Nivaldo D Costa \\ Embrapa Semiárido, C. Postal 23, 56300-000 Petrolina-PE; geraldo.milanez@embrapa.br; nivaldo.costa@embrapa.br
}

\section{RESUMO}

Os elementos mais absorvidos em termos de porcentagem na matéria seca da cebola são o potássio e nitrogênio. Com o objetivo de avaliar a produtividade e armazenamento pós-colheita de cultivares de cebola, em função de doses de nitrogênio foi realizado um experimento no período de setembro 2009 a fevereiro de 2010 . O delineamento experimental utilizado foi de blocos ao acaso no esquema fatorial $5 \times 2$, compreendendo cinco doses de nitrogênio $(0 ; 60 ; 120,180$ e 240 $\mathrm{kg} \mathrm{ha}^{-1}$ ) e duas cultivares (Alfa Tropical e Alfa São Francisco), com três repetições. Maiores produtividades comerciais foram obtidas nas doses de 161,4 $\mathrm{kg} \mathrm{ha}^{-1}$ de N (Alfa Tropical) e 215,9 $\mathrm{kg} \mathrm{ha}^{-1}$ de N (Alfa São Francisco) associadas às doses mais econômicas de 159,6 e 213,8 $\mathrm{kg} \mathrm{ha}^{-1}$ de N, respectivamente. Com o aumento das doses de $\mathrm{N}$, houve redução da produção de bulbos não comerciais ou refugos, sendo a menor produção de refugos estimada na dose de $185,4 \mathrm{~kg} \mathrm{ha}^{-1}$ de $\mathrm{N}$. Bulbos comerciais de maior massa fresca e diâmetro foram obtidos com o incremento das doses de nitrogênio; no entanto, aos 60 dias após cura, detectaram-se efeitos quadráticos com pontos de máxima perda de massa na dose de $149,0 \mathrm{~kg} \mathrm{ha}^{-1}$ de $\mathrm{N}(38,4 \%)$.

Palavras-chave: Allium cepa, rentabilidade, nutrição vegetal, perda de massa fresca.

\begin{abstract}
Nitrogen levels on yield and postharvest storage of onion cultivars

The elements absorbed as a percentage of dry matter in onions are potassium and nitrogen. One trial was carried out to evaluate the influence of nitrogen levels and cultivars on yield and postharvest storage from September 2009 to February 2010. The experimental design was a completely randomized block in a $5 \times 2$ factorial scheme, composed of five nitrogen levels $\left(0 ; 60 ; 120,180\right.$ and $\left.240 \mathrm{~kg} \mathrm{ha}^{-1}\right)$ and two cultivars (Alfa Tropical and Alfa São Francisco) with three replications. Highest commercial yields were obtained at doses of $161.4 \mathrm{~kg} \mathrm{ha}^{-1} \mathrm{~N}$ (Alfa Tropical) and $216.2 \mathrm{~kg} \mathrm{ha}^{-1} \mathrm{~N}$ (Alfa São Francisco) associated with economic doses of 159.6 and $213.8 \mathrm{~kg}$ $\mathrm{ha}^{-1}$ of $\mathrm{N}$ respectively. With increasing $\mathrm{N}$ levels, a gradual reduction in the production of non-commercial bulbs was verified, and a lower production of refuse was obtained when an estimated dose of 185.4 $\mathrm{kg} \mathrm{ha}^{-1}$ of $\mathrm{N}$ was applied. Commercial bulbs of higher fresh mass and diameter were obtained with the increment of nitrogen. However, after 60 days of curing we detected quadratic effects with maximum fresh loss of bulb at the dose of $149.0 \mathrm{~kg} \mathrm{ha}^{-1} \mathrm{~N}(38.4 \%)$.
\end{abstract}

Keywords: Allium cepa, profitability, plant nutrition, loss of fresh mass.

(Recebido para publicação em 23 de setembro de 2013; aceito em 4 de junho de 2014) (Received on September 23, 2013; accepted on June 4, 2014)

$\mathrm{N}_{\mathrm{c}}^{\circ}$ o Brasil, a cebola (Allium cepa) é considerada a terceira hortaliça mais importante em termos de valor econômico, suplantada apenas pelo tomate e batata, sendo consumida in natura em saladas ou como condimento. Em 2012, a produtividade média nacional se situou em torno de 24,7 $\mathrm{t} \mathrm{ha}^{-1}$, sendo que nos estados de Pernambuco e Bahia, maiores produtores do Nordeste alcançaram produtividade média de 29,0 e 20,4 t ha-1, respectivamente (IBGE, 2013).

$\mathrm{O}$ nitrogênio $(\mathrm{N})$ contribui marcadamente para a melhoria da produção de cebola, sendo absorvido em grandes quantidades e superado somente pelo potássio (May et al., 2008; Vidigal, 2010).
Em geral, a demanda em $\mathrm{N}$ pela cultura da cebola é grande a partir da metade do ciclo. É muito pequena nos primeiros 60 dias após a semeadura e a partir de então a demanda aumenta. Porém, este elemento é muito importante na fase inicial do desenvolvimento da planta da cebola, para a formação do sistema radicular e emissão de folhas (Porto et al., 2007; Cecílio Filho et al., 2009).

Quanto à capacidade de resposta dessa cultura a doses de nitrogênio, vários autores relatam que este nutriente contribui marcadamente para uma melhor produtividade. Sua produtividade em bulbos respondeu significativamente à aplicação de nitrogênio até $100 \mathrm{~kg} \mathrm{ha}^{-1}$
(Yadav et al., 2003), $249 \mathrm{~kg} \mathrm{ha}^{-1}$ de $\mathrm{N}$ (Kurtz et al., 2012), $263 \mathrm{~kg} \mathrm{ha}^{-1}$ (Boyhan et al., 2007) e $300 \mathrm{~kg} \mathrm{ha}^{-1}$ (Zeca et al., 2009).

Em relação ao armazenamento pós-colheita, Singh \& Dhankhar (1991) verificaram, em bulbos armazenados em condições de ambiente, que a perda de massa e a incidência de bulbos podres e brotados aumentaram com o incremento das doses de nitrogênio (80 a $160 \mathrm{~kg}$ $\mathrm{ha}^{-1}$ de $\mathrm{N}$ ) e reduziram com o acréscimo de $100 \mathrm{~kg} \mathrm{ha}^{-1}$ de $\mathrm{K}_{2} \mathrm{O}$. Segundo esses autores, a dose de $80 \mathrm{~kg} \mathrm{ha}^{-1}$ de $\mathrm{N}$ mais $100 \mathrm{~kg} \mathrm{ha}^{-1}$ de $\mathrm{K}_{2} \mathrm{O}$ levaram às melhores respostas no armazenamento. No entanto, Hussaini et al. (2000) não observaram efeitos do nitrogênio até a 
dose de $164 \mathrm{~kg} \mathrm{ha}^{-1}$, dezenove semanas após a colheita. Avaliando as perdas pós-colheita aos 135 dias de armazenagem, Kurtz et al. (2012) constataram aumento com o incremento da dose de nitrogênio na safra 2008/09, que não foram influenciadas pela adição de $\mathrm{N}$ na safra seguinte. Em 2008/09, as perdas passaram de $19,5 \%$, na testemunha, para um máximo de $34,5 \%$, que ocorreu com a aplicação de $158 \mathrm{~kg} \mathrm{ha}^{-1}$ de N. Na safra 2009/10, as perdas pós-colheita não foram influenciadas pela adição de $\mathrm{N}$ e atingiram, em média, 20,8\%.

Segundo Raij (1991), a lei dos incrementos decrescentes serve de base para a definição da dose mais econômica de um insumo. Quando o valor do aumento em produção é igual ao custo do fertilizante, atinge-se um nível de aplicação acima do qual não se traduz em retorno econômico e corresponde à chamada dose econômica. Na definição da dose de nutriente a ser aplicada, a meta deve ser sempre a de se utilizar doses econômicas do fertilizante, o que irá depender em parte da razão entre o preço do produto agrícola e o do fertilizante, e também do tipo de resposta ao nutriente na área específica (Prochnow, 2008).

Nesse contexto, o objetivo desse trabalho foi avaliar o efeito de doses de nitrogênio sobre a produtividade e armazenamento pós-colheita de cultivares de cebola, calculando-se a dose economicamente viável.

\section{MATERIAL E MÉTODOS}

O experimento foi conduzido no período de setembro 2009 a fevereiro de 2010, em Petrolina-PE (9'9'S, 40²9'O, 365,5 m de altitude). Segundo a classificação climática de Köppen, a região apresenta clima do tipo BSWh', semiárido, com os valores médios anuais das variáveis climatológicas: temperatura do ar $=26,5^{\circ} \mathrm{C}$, precipitação pluvial $=541,1 \mathrm{~mm}$, umidade relativa do $\mathrm{ar}=$ $65,9 \%$, evaporação do tanque classe " $\mathrm{A}$ " $=2.500 \mathrm{~mm}$ ano $^{-1}$ e velocidade do vento $=2,3 \mathrm{~m} \mathrm{~s}^{-1}$. A precipitação é irregularmente distribuída no espaço e no tempo, concentrando-se nos meses de dezembro a abril e a insolação anual é superior a 3.000 h (Azevedo et al., 2006). O solo foi classificado como Latossolo Vermelho Amarelo Distróferrico (Santos et al., 2006), apresentou $\mathrm{pH}\left(\mathrm{H}_{2} \mathrm{O}\right)=6,0 ; \mathrm{Ca}=$ $2,1 \mathrm{cmol}_{\mathrm{c}} \mathrm{dm}^{-3} ; \mathrm{Mg}=0,8 \mathrm{cmol}_{\mathrm{c}} \mathrm{dm}^{-3} ; \mathrm{Na}=$ $0,01 \mathrm{cmol}_{\mathrm{c}} \mathrm{dm}^{-3} ; \mathrm{K}=0,38 \mathrm{cmol}_{\mathrm{c}} \mathrm{dm}^{-3} ; \mathrm{Al}=$ $0,00 \mathrm{cmol}_{\mathrm{c}} \mathrm{dm}^{-3}, \mathrm{P}($ Mehlich $)=13,0 \mathrm{mg}$ $\mathrm{dm}^{-3} \mathrm{e} \mathrm{MO}=3,0 \mathrm{~g} \mathrm{~kg}^{-1}$.

$\mathrm{O}$ delineamento experimental utilizado foi de blocos ao acaso no esquema fatorial $5 \times 2$, compreendendo cinco doses de nitrogênio $(0 ; 60 ; 120,180$ e $240 \mathrm{~kg} \mathrm{ha}^{-1}$ ) e duas cultivares (Alfa Tropical e Alfa São Francisco), com três repetições.

A parcela experimental constou de oito linhas de $3,0 \mathrm{~m}$ de comprimento, espaçadas de $0,15 \mathrm{~m}$, com $0,10 \mathrm{~m}$ entre plantas, perfazendo uma área total de 3,6 $\mathrm{m}^{2}$ (3,0 x 1,2 m), sendo utilizadas como área útil as seis linhas centrais, retirando-se $0,50 \mathrm{~m}$ em cada extremidade $\left(1,80 \mathrm{~m}^{2}\right)$. A adubação constou da aplicação de $134,0 \mathrm{~kg}$ de $\mathrm{P}_{2} \mathrm{O}_{5}$ ha $^{-1}$ no plantio (Cavalcanti, 2008) e $90 \mathrm{~kg}$ $\mathrm{ha}^{-1}$ de $\mathrm{K}_{2} \mathrm{O}$ ha $^{-1}$ divididos no plantio e em cobertura (Resende et al., 2008), tendo como suas fontes o superfosfato simples e cloreto de potássio. As adubações nitrogenada e potássica foram divididas em três parcelamentos, sendo a primeira realizada no plantio (1/3) e o restante $(2 / 3)$ em duas coberturas aos 25 e 50 dias após transplantio. Como fonte de nitrogênio utilizou-se ureia, que foi aplicada em sulcos lateralmente às linhas de plantio, e coberta com solo.

O transplante das mudas ocorreu em setembro aos 30 dias de idade e o preparo do solo constou de aração, gradagem e levantamento dos canteiros a 0,20 m de altura. As irrigações foram feitas através do método de microaspersão, com turno de dois dias e lâminas de água em torno de $10 \mathrm{~mm}$, calculada em função da evaporação do tanque classe A, e os tratos fitossanitários foram os comuns à cultura da cebola.

As colheitas foram realizadas em dezembro quando as plantas apresentaram sinais avançados de senescência, como amarelecimento e seca das folhas e quando mais de $70 \%$ das plantas se encontravam estaladas. A cura foi realizada ao sol por três dias e 12 dias à sombra, em galpão ventilado.

Avaliou-se a produtividade comercial em bulbos (bulbos perfeitos e com diâmetro transversal acima de 35 $\mathrm{mm}$ ) e a não comercial ou de refugos (bulbos com diâmetro inferior a 35 $\mathrm{mm}$ ), expressas em $\mathrm{t} \mathrm{ha} \mathrm{h}^{-1}$, aos 15 dias após a cura (Filgueira, 2008). A massa fresca de bulbos ( $\mathrm{g}$ bulbo $^{-1}$ ) foi determinada dividindo-se o peso de bulbos comerciais após a cura pelo número de bulbos. Após o período de cura, os bulbos foram armazenados à temperatura ambiente e realizadas pesagens aos $30 \mathrm{e}$ 60 dias após a colheita, sendo os valores comparados àqueles obtidos ao final da cura (15 dias após colheita). Os valores foram transformados em percentagem de perda de massa. Os bulbos comerciais (\%) foram classificados segundo o diâmetro transversal ( $\mathrm{mm}$ ) em classe 2 (>35-50 mm); classe 3 (>50-70 $\mathrm{mm})$; classe $4(>70-90 \mathrm{~mm})$ e classe $5(>90$ $\mathrm{mm}$ ) (Resende et al., 2008). Os dados de porcentagem foram transformados em arco-seno $\sqrt{P / 100}$ para efeitos de análise estatística.

Também foi determinada a dose mais econômica de nitrogênio para a produtividade de bulbos de cebola, conforme Raij (1991) e Natale et al. (2011). Neste estudo, considerou-se o preço médio por $\mathrm{kg}$ de bulbo de cebola comercializado no Mercado do Produtor de Juazeiro-BA, no valor de R\$1,24, referente ao ano de 2012 (SEAGRI, 2013). $\mathrm{O}$ custo do $\mathrm{kg}$ de nitrogênio, cuja fonte foi a ureia no mercado local foi $\mathrm{R} \$ 3,11$ (por kg do elemento). Dessa maneira, a "moeda" utilizada nos cálculos, durante todo o estudo, foi a própria cebola, considerando-se a seguinte relação de equivalência: kg de nitrogênio aplicado/ $\mathrm{kg}$ de cebola comercializada no Mercado do Produtor de Juazeiro-BA igual a $\mathrm{R} \$ 3,11 / \mathrm{R} \$ 1,24=\mathrm{R} \$ 2,50$.

As doses mais econômicas foram calculadas com base na derivada da equação de regressão entre a produção de bulbos e as doses de nitrogênio aplicadas, tornando-a igual à relação de troca, ou seja: $d y / d x=a_{1}+2 a_{2} x=$ relação de troca. A dose mais econômica $(x)$ foi calculada por: $x^{1}=\left(A_{1}\right.$ - relação de troca $) / 2$. $\left(-\mathrm{A}_{2}\right)$

Os dados coletados foram submetidos à análise de variância e regressão com base no modelo polinomial, utilizando-se o teste F para comparação dos quadrados médios a $5 \%$ de proba- 
bilidade. As doses de nitrogênio foram ajustadas a equações de regressão polinomiais adotando-se como critério para escolha do modelo, o efeito significativo pelo teste de $\mathrm{F}$ a $5 \%$ de probabilidade e a magnitude dos coeficientes de determinação, empregando-se o programa SISVAR 5.0 (Ferreira, 2010).

\section{RESULTADOS E DISCUSSÃO}

Os resultados evidenciaram efeitos significativos independentes para as doses de nitrogênio $(\mathrm{N})$ e cultivares de cebola, assim como para sua interação, variando com as características avaliadas.

Para a produtividade comercial verificou-se efeitos significativos da interação entre doses de nitrogênio e cultivares (Figura 1). Para a cultivar Alfa Tropical, a dose de $161,4 \mathrm{~kg} \mathrm{ha}^{-1}$ de $\mathrm{N}$ promoveu a maior produtividade comercial $\left(69,20\right.$ t ha $\left.^{-1}\right)$, enquanto para a cultivar Alfa São Francisco se estimou a dose de 215,9 kg ha-1 de N (65,05 t ha-1) (máxima eficiência física do insumo). Estes resultados estão coerentes aos observados por diferentes autores que obtiveram respostas positivas da aplicação de $\mathrm{N}$ na cultura da cebola (Neeraja et al., 2001; Diaz-Perez et al., 2003; Singh et al., 2004), no entanto, inferiores aos relatados por Boyhan et al. (2007) que observaram a melhor resposta com a dose de $263,0 \mathrm{~kg} \mathrm{ha}^{-1}$ de $\mathrm{N}$ e aos de Vidigal (2000) que obtiveram as doses de 261,0 e 265,0 kg de $\mathrm{N} \mathrm{ha}^{-1}$, como as que proporcionaram a máxima produção de bulbos comercializáveis, em cultivo de inverno e de verão, respectivamente.

Utilizando-se a equação de regressão da Figura $1(\mathrm{Y}=50.753+228,6111 \mathrm{X}$ - 0,708333 $\mathrm{X}^{2}$ ) pode-se calcular a dose mais econômica de nitrogênio para a maior produtividade de bulbos da cultivar Alfa Tropical:

$x^{1}=(228,6111-2,50) / 2 .(0,708333)$ $=159,6 \mathrm{~kg} \mathrm{ha}^{-1}$

A receita prevista, decorrente da adubação nitrogenada, pode ser determinada pelo aumento de produção de bulbos $=18,44 \mathrm{t} /$ ha (produção de bulbos com a dose mais econômica de $\mathrm{N}$ igual a 69,20 t ha-1 menos a produção na dose zero de $\mathrm{N}$ igual a 50,75 $\mathrm{t} \mathrm{ha}^{-1}$ ).

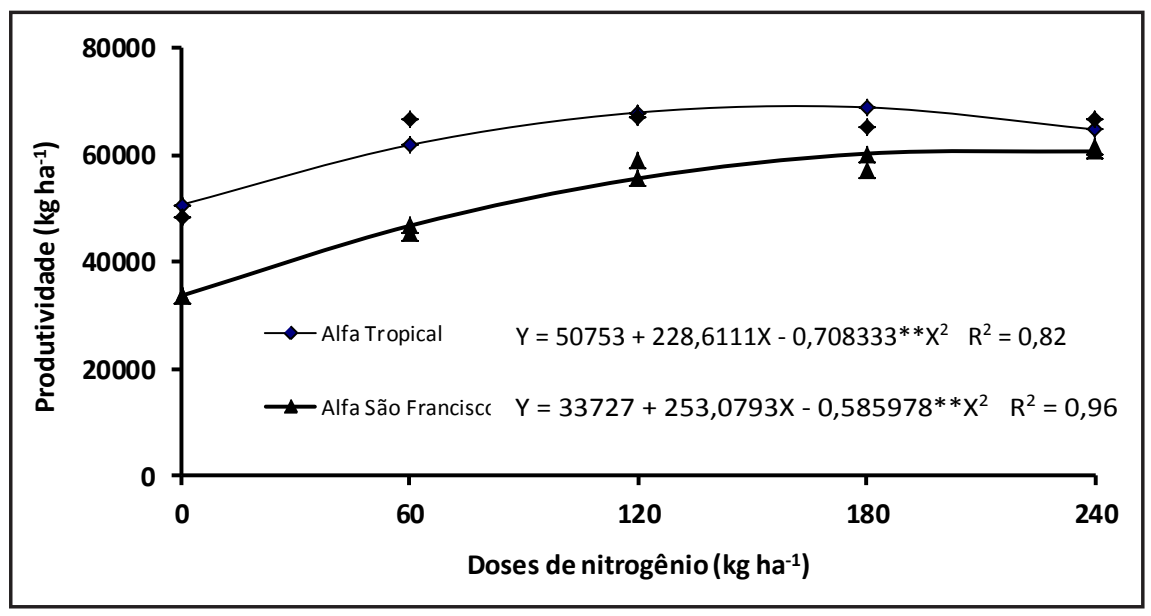

Figura 1. Produtividade das cultivares Alfa tropical e Alfa São Francisco em função de doses de nitrogênio (yield of onion cultivars depending on nitrogen levels). Petrolina, Embrapa Semiárido, 2009/2010.

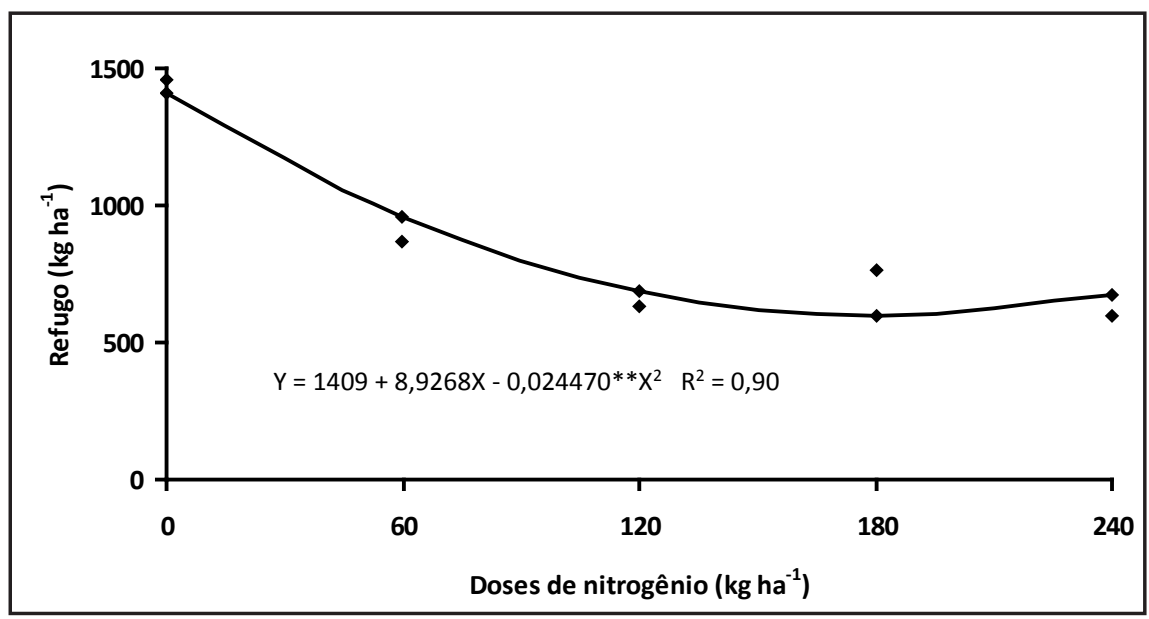

Figura 2. Produtividade não comercial de bulbos (refugos) de duas cultivares de cebola em função de doses de nitrogênio (non-commercial bulbs yield of two cultivars depending on nitrogen levels). Petrolina, Embrapa Semiárido, 2009/2010.

Subtraindo-se o custo do adubo nitrogenado, em quilogramas de bulbo $(156,6$ $\mathrm{kg} \times \mathrm{R} \$ 3,11=496,35 / \mathrm{R} \$ 1,24=400,1$ $\mathrm{kg} \mathrm{ha}^{-1}$ ), obteve-se uma receita prevista de 18,04 tha-1 de bulbos comerciais, ou seja, uma receita liquida da ordem de $\mathrm{R} \$ 22.373,20$.

Utilizando-se a mesma sistemática pode-se calcular a dose mais econômica para a cultivar Alfa São Francisco, que foi de $213,8 \mathrm{~kg} \mathrm{ha}^{-1}$. A receita prevista, decorrente da adubação nitrogenada foi 30,64 $\mathrm{t} \mathrm{ha}^{-1}$ de bulbos comerciais, ou seja, uma receita liquida da ordem de $\mathrm{R} \$ 37.999,20$.

Um resumo das doses mais econômicas do nitrogênio em função da produtividade e custos de cultivares de cebola é apresentado na Tabela 1. É importante destacar, que as produtividades obtidas com as diferentes doses econômicas estiveram muito próximas das produtividades máximas possíveis, com 99\%, para as duas cultivares avaliadas. Desse modo, a aplicação das doses mais econômicas permitiu economia na aplicação de nitrogênio, sem perda significativa na produtividade de bulbos.

Analisando-se as doses econômicas obtidas em uma relação lucro versus doses recomendadas (Tabela 1), a cultivar Alfa Tropical obteria um índice $(18.042,9 / 159,6)$ de $113,0 \mathrm{~kg}$ de bulbos por $\mathrm{kg}$ de nitrogênio aplicado, enquanto a cultivar Alfa São Francisco $(30.644,5 / 213,8)$ alcançaria $143,3 \mathrm{~kg}$ de bulbos por $\mathrm{kg}$ de nitrogênio aplicado. Estes resultados poderiam ser justificados 
em função da maior ou menor eficiência das cultivares na absorção do nitrogênio, assimilação e transformação em massa fresca de bulbo, como também pelo bom preço alcançado pela cebola no período. May et al. (2008), comparando as cultivares Optima e Superex, observaram em 'Superex' extrações de $\mathrm{N}$ menores e maior produtividade que a 'Optima', sugerindo maior eficiência na utilização do nutriente, como os resultados obtidos no presente estudo para a cultivar Alfa São Francisco.

Não se verificou para produção de refugos (bulbos não comerciais) efeitos da interação. A cultivar Alfa Tropical com $0,95 \mathrm{t} \mathrm{ha}^{-1}$ mostrou valores maiores que os obtidos pela cultivar Alfa São Francisco, com 0,78 $\mathrm{t} \mathrm{ha}^{-1}$ (Tabela 2). Verificou-se com o aumento das doses uma redução gradativa na produção de bulbos considerados não comerciais, sendo a menor produção de refugos estimada na dose de $182,4 \mathrm{~kg} \mathrm{ha}^{-1}$ de $\mathrm{N}$ (Figura 2). Estes resultados mostram a capacidade de resposta da cebola à aplicação de nitrogênio e alicerça as afirmações de vários autores, ou seja, que esse elemento contribui marcadamente para uma melhor produtividade da cultura, sobretudo, na produção de bulbos de maior tamanho (Ghaffoor et al., 2003; Lee et al., 2003; Mandira \& Khan, 2003).

Os resultados positivos da adubação nitrogenada alcançados no presente trabalho se devem ao seu papel funcional no metabolismo de desenvolvimento da cebola. $\mathrm{O}$ nitrogênio é encontrado em compostos orgânicos como aminoácidos e ácidos nucléicos e participa de diversos processos fisiológicos vitais para o ciclo de vida das plantas (absorção iônica, fotossíntese, respiração, multiplicação e diferenciação celulares e herança) (Mengel \& Kirkby, 2001; Epstein \& Bloom, 2006). A sua deficiência retarda o crescimento e desenvolvimento da planta, com consequente redução da qualidade e produtividade da cultura. Broadley et al. (2000) relatam uma relação negativa entre plantas em condições normais de nitrogênio disponível e plantas deficientes, ocorrendo redução na massa fresca das folhas, em condições de limitação do nutriente.

Resultados similares foram observa-

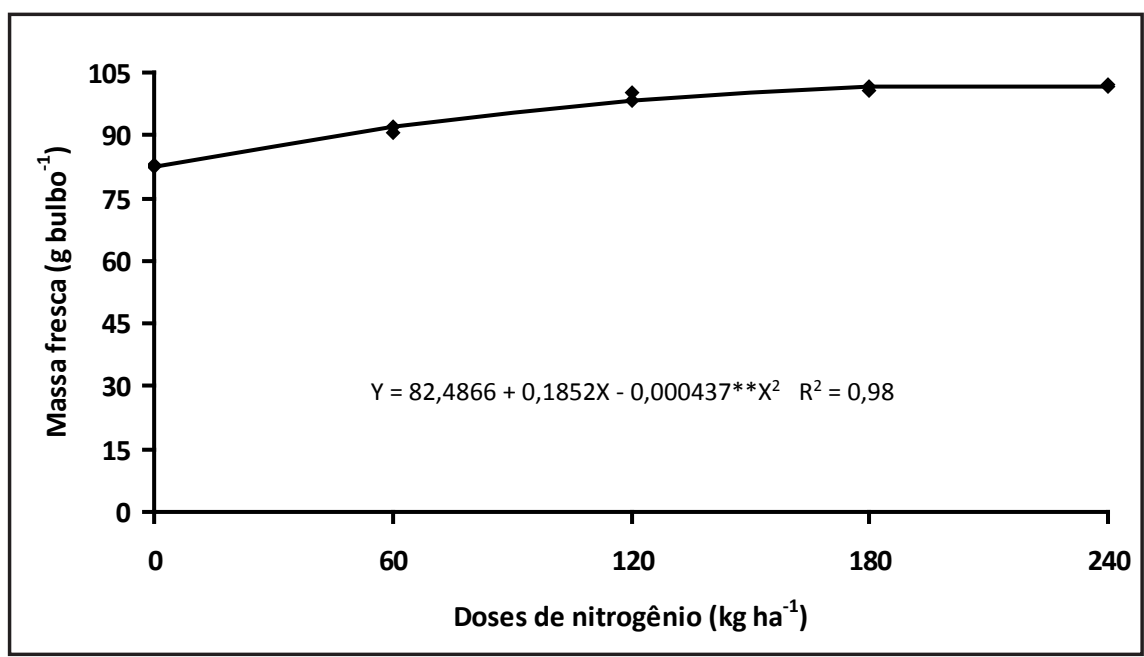

Figura 3. Massa fresca de bulbos de duas cultivares de cebola em função das doses de nitrogênio (fresh mass of bulbs of two onion cultivars depending on nitrogen levels). Petrolina, Embrapa Semiárido, 2009/2010.

dos para a massa fresca de bulbo, mas sem efeitos da interação, verificando-se para a cultivar Alfa Tropical maior valor $\left(99,7 \mathrm{~g} \mathrm{bulbo}^{-1}\right)$ que para a cv. Alfa São Francisco (90,8 $\mathrm{g} \mathrm{bulbo}^{-1}$ ) (Tabela 2). Quanto ao efeito de doses, os bulbos de maior massa fresca foram obtidos com 211,9 kg ha-1 de N (Figura 3). Diversos autores corroboram estes resultados informando resposta positiva do $\mathrm{N}$ no aumento da massa fresca do bulbo (Hussaini et al., 2000; Lee et al., 2003; Mandira \& Khan, 2003; Cecílio Filho et al., 2010).

A conservação pós-colheita apresentou resultados diferentes das doses de $\mathrm{N}$ e das cultivares, em função do período de armazenagem (Tabela 2). Para perda de massa não se constatou efeitos significativos aos 30 dias, ocorrendo para a cultivar Alfa Tropical perdas da ordem de $14,3 \%$ e para a cv. Alfa São Francisco, 15,5\%, verificando-se para as doses uma variação entre 12,9 e $18,2 \%$. No que se refere à perda de massa fresca dos bulbos aos 60 dias após a cura, detectou-se efeitos quadráticos com pontos de máxima perda na dose de $149,0 \mathrm{~kg} \mathrm{ha}^{-1}$ de $\mathrm{N}$, ou $38,4 \%$ (Tabela 2 ), observando-se menor perda $(33,8 \%)$ na ausência de adubação.

Uma maior conservação pós-colheita apresenta relevante importância por ocasião da comercialização do produto, que apresenta grandes variações de preços, em curtos períodos de tempo. $\mathrm{O}$ produtor, em função do conhecimen- to das perdas de massa pela cebola, poderá alcançar melhores cotações de preços e incrementar ou maximizar seus lucros, em função da volatilidade de seu mercado. Os resultados obtidos em pós-colheita são comparáveis aos encontrados por Kurtz et al. (2012) que verificaram perdas aos 135 dias após a colheita, de 19,5\% na testemunha, para um máximo de $34,5 \%$, quando ocorreu a aplicação de $158 \mathrm{~kg} \mathrm{ha}^{-1}$ de N. Todavia, Hussaini et al. (2000) não observaram efeitos do nitrogênio até a dose de $164 \mathrm{~kg} \mathrm{ha}^{-1}$, dezenove semanas após a colheita.

A classificação de bulbos comerciais de cebola registrou efeitos da interação (Tabela 2), para as diferentes classes. No que se refere à classificação de bulbos classe 2 que são bulbos de tamanho inferior (>35-50 mm de diâmetro), a cultivar Alfa São Francisco apresentou redução linear com o incremento das doses de $\mathrm{N}$, enquanto a dose de $179,9 \mathrm{~kg} \mathrm{ha}^{-1}$ de $\mathrm{N}$ propiciaram a menor produção de bulbos nessa classe para a cultivar Alfa Tropical. Para a classe 3 (bulbos maiores, $>50-70 \mathrm{~mm}$ de diâmetro), a dose de 149,0 $\mathrm{kg} \mathrm{ha}^{-1}$ de $\mathrm{N}$ promoveu a maior produção para a cultivar Alfa Tropical, e redução linear com o incremento das doses de nitrogênio para a cultivar Alfa São Francisco (Tabela 2). Resende et al. (2008) verificaram que a aplicação de nitrogênio reduziu gradativamente a produção de bulbos refugo (não comerciais) e elevou para até $85,8 \%$ a 
Tabela 1. Dose econômica de nitrogênio em função da produtividade e custos de cultivares de cebola (economic levels of nitrogen depending on yield and costs for onion cultivars). Petrolina, Embrapa Semiárido, 2009/2010.

\begin{tabular}{lcccc}
\hline Cultivar & $\begin{array}{c}\text { Aumento de } \\
\text { produção (t/ha) }\end{array}$ & $\begin{array}{c}\text { Custo do nitrogênio } \\
\text { (kg bulbo/ha) }\end{array}$ & $\begin{array}{c}\text { Lucro } \\
\text { (kg bulbo/ha) }\end{array}$ & $\begin{array}{c}\text { Produtividade }^{\mathbf{1}} \\
\mathbf{( \% )}\end{array}$ \\
\hline Alfa Tropical & 18,44 & 400,1 & $18.042,9$ & 99 \\
Alfa São Francisco & 31,47 & 824,5 & $30.644,5$ & 99 \\
\hline
\end{tabular}

${ }^{1}$ Porcentagem da produtividade de bulbos obtida com a dose mais econômica, em relação à produtividade máxima (bulb yield obtained with the economic level of nitrogen in percentage of the highest yield).

Tabela 2. Produtividade não comercial (refugos), massa fresca do bulbo, perda de massa fresca e classificação, em classes, segundo o diâmetro transversal de bulbos de duas cultivares de cebola em função de doses de nitrogênio (N) [non-commercial bulb yield, fresh mass of bulb, fresh mass loss and classification in classes (\%) according to the transversal diameter of two onion bulbs cultivars depending on nitrogen levels (N)]. Petrolina, Embrapa Semiárido, 2009/2010.

\begin{tabular}{|c|c|c|}
\hline Características & Alfa Tropical & Alfa São Francisco \\
\hline Refugos (t/ha) & $0,95 \mathrm{a}$ & $0,78 \mathrm{~b}$ \\
\hline Massa fresca (g/bulbo) & $99,70 \mathrm{a}$ & $90,80 \mathrm{~b}$ \\
\hline Perda de massa 60 DAC (\%) & $31,90 \mathrm{~b}$ & $39,60 \mathrm{a}$ \\
\hline & Equações de regressão & $\mathbf{R}^{2}$ \\
\hline Perda de massa 60 DAC (\%) & $\mathrm{N}: \mathrm{Y}=33,7849+0,0617 \mathrm{X}-0,000207 * \mathrm{X}^{2}$ & 0,74 \\
\hline Classe $2(\%)$ & $\begin{array}{l}\mathrm{N}: \text { Alfa Tropical } Y=35,3423-0,1450 \mathrm{X}+0,000403 * * \mathrm{X}^{2} \\
\mathrm{~N}: \text { Alfa } \mathrm{SF} Y=37,3047-0,0813^{* *} \mathrm{X}\end{array}$ & $\begin{array}{l}0,94 \\
.0,96\end{array}$ \\
\hline Classe $3(\%)$ & $\begin{array}{l}\mathrm{N}: \text { Alfa Tropical } \mathrm{Y}=51,7967+0,1203 \mathrm{X}-0,000405^{* *} \mathrm{X}^{2} \\
\mathrm{~N}: \text { Alfa } \mathrm{SF} Y=51,7492-0,0701^{* * X}\end{array}$ & $\begin{array}{l}0,94 \\
.0,95\end{array}$ \\
\hline Classe $4(\%)$ & $\begin{array}{l}\mathrm{N}: \text { Alfa Tropical } \mathrm{Y}=11,7086+0,0360 * * \mathrm{X} \\
\mathrm{N}: \text { Alfa } \mathrm{SF} Y=7,7317+0,0183 * * \mathrm{X}\end{array}$ & $\begin{array}{l}0,96 \\
0,74\end{array}$ \\
\hline
\end{tabular}

Médias seguidas pela mesma letra, na linha, não diferem entre si, pelo teste de F, $5 \%$ (average values followed by the same letter in the lines do not differ significantly, according to $\mathrm{F}$ test $\mathrm{p}<0.05)$. **, ${ }^{*}$ Significativo a 1 e $5 \%$ de probabilidade, pelo teste de $\mathrm{F}(* *, *$ significant at 1 and $5 \%$ level of probability by the $\mathrm{F}$ test, respectively).

quantidade de bulbos distribuídos entre 50 e $70 \mathrm{~mm}$ de diâmetro com a dose estimada de $153,6 \mathrm{~kg} \mathrm{ha}^{-1} \mathrm{de} \mathrm{N}$, retratando, dessa forma, que esse nutriente contribui muito para a melhoria da produtividade da espécie estudada.

Com relação à classe 4 (bulbos de maior calibre, $>70-90 \mathrm{~mm}$ de diâmetro) os resultados demonstraram efeitos lineares positivos das cultivares, com o aumento das doses de N. May et al. (2007) e Resende et al. (2008) também obtiveram bulbos de maior diâmetro quando aumentaram as doses de N. A obtenção de bulbos maiores, além de estar diretamente relacionada com o aumento no rendimento, também aumenta a lucratividade, pois bulbos com diâmetro inferior a $50 \mathrm{~mm}$ apresentam menor valor de mercado do que bulbos maiores (Kurtz et al., 2012) das classes 3 e 4, que alcançam melhores preços de mercado (Vidigal, 2000).

Os resultados obtidos permitem concluir que o incremento das doses de $\mathrm{N}$ promoveu redução gradativa na produção de refugos, bulbos comerciais de maior massa fresca e diâmetro. Não se verificou perda de massa significativa aos 30 dias após a cura com as doses de $\mathrm{N}$; no entanto, aos 60 dias após a cura, detectaram-se efeitos quadráticos com pontos de máxima perda $(38,4 \%)$, na dose de 149,0 kg ha-1 de N. Conforme preconizado na Lei dos Incrementos Decrescentes (Raij, 1991), a adubação correta a ser aplicada é a que proporciona maior lucro para o produtor. Nesse contexto, a aplicação das doses mais econômicas são $159,6 \mathrm{~kg} \mathrm{ha}^{-1} \mathrm{de}$ N para a cultivar Alfa Tropical e 213,8 ha $^{-1}$ de N para a cv. Alfa São Francisco, para as condições de cultivo no verão, no Submédio do Vale do São Francisco.

\section{AGRADECIMENTOS}

Os autores agradecem ao CNPq pelo auxílio à pesquisa concedido (Proc.
474186/2006-7).

\section{REFERÊNCIAS}

AZEVEDO PV; SOUSA IF; SILVA BB; SILVA VPR. 2006. Water-use efficiency of dwarfgreen coconut (Cocos nucifera) orchards in Northeast Brazil. Agricultural Water Management 1: 259-264.

BOYHAN GE; TORRANCE RL; HILL CR. 2007. Effects of nitrogen, phosphorus, and potassium rates and fertilizer sources on yield and leaf nutrient status of short-day onions. HortScience 42: 653-660.

BROADLEY MR; ESCOBAR-GUTIERREZ AJ; BURNS AJ; BURNS IG. 2000. What are the effects of nitrogen deficiency on growth components of lettuce?. New Phytologist 3: 519-526.

CAVALCANTI FJA. (coord). 2008. Recomendações de adubação para o Estado de Pernambuco: $2^{\mathrm{a}}$ aproximação, 3 ed. Recife: IPA. 212 p.

CECÍLIO FILHO AB; MARCOLINI MW; MAY A; BARBOSA JC. 2010. Produtividade e classificação de bulbos de cebola em função da fertilização nitrogenada e potássica, em semeadura direta. Cientifica 38: 14-22. 
CECÍLIO FILHO AB; MAY A; PÔRTO DRQ; BARBOSA JC. 2009. Crescimento da cebola em função de doses de nitrogênio, potássio e da população de plantas em semeadura direta. Horticultura Brasileira 27: 49-54.

DIAZ-PEREZ JC; PURVIS AC; PAULK JT. 2003. Bolting, yield, and bulb decay of sweet onion as affected by nitrogen fertilization. Journal of the American Society Horticultural Science 128: 144-149.

EPSTEIN E; BLOOM AJ. 2006. Nutrição mineral de plantas: princípios e perspectivas. 2. ed. Londrina: Planta, 403p.

FERREIRA DF. 2010. SISVAR Versão 5.3. Lavras: Departamento de Ciências Exatas, UFLA.

FILGUEIRA FAR. 2008. Novo manual de olericultura: agrotecnologia moderna na produção e comercialização de hortaliças. Viçosa: Editora UFV, 3. ed. 421p.

GHAFFOOR A; JILANI MS; KHALIQ G; WASEEM K. 2003. Effect of different NPK levels on the yield of three onion (Allium cepa) varieties. Asian Journal Plant Sciences 2: 342-346.

HUSSAINI MA; AMANS EB; RAMALAN AA. 2000. Yield, bulb size distribution, and storability of onion (Allium cepa) under different levels of $\mathrm{N}$ fertilization and irrigation regime. Tropical Agriculture 77: 145-149.

IBGE. 2013. Levantamento Sistemático da Produção Agrícola. Rio de Janeiro: IBGE. 26: $1-84$.

KURTZ C; ERNANI PR; COIMBRA JLM; PETRY E. 2012. Rendimento e conservação de cebola alterados pela dose e parcelamento de nitrogênio em cobertura. Revista Brasileira de Ciência do Solo 36: 865-876.

LEE JT; HA IJ; LEE CJ; MOON JS; CHO YC. 2003. Effect of $\mathrm{N}, \mathrm{P}_{2} \mathrm{O}_{5}$, and $\mathrm{K}_{2} \mathrm{O}$ application rates and top dressing time on growth and yield of onion (Allium cepa) under spring culture in lowland. Korean Journal Horticultural Science and Technology 21: 260-266.

MANDIRA C; KHAN AH. 2003. Effect of nitrogen and potassium on growth, yield and yield attributes of onion. New Agriculturist 14: 9-11.

MAY A; CECÍLIO FILHO AB; PORTO DRQ; VARGAS PF; BARBOSA CB. 2007. Produtividade de híbridos de cebola em função da população de plantas e da fertilização nitrogenada e potássica. Horticultura Brasileira 25: 53-59.

MAY A; CECÍLIO FILHO AB; PORTO DRQ; VARGAS PF; BARBOSA JC. 2008. Acúmulo de macronutrientes por duas cultivares de cebola produzidas em sistema de semeadura direta. Bragantia, 67: 507-512.

MENGEL K; KIRKBY EA. 2001. Principles of plant nutrition. 5. ed. Dordrecht: Kluwer Academic Publishers. 849p.

NATALE W; ROZANE DE; PRADO RM; ROMUALDO LM; SOUZA HAS; HERNANDES A. 2011. Dose econômica de calcário na produtividade de caramboleiras. Revista Brasileira de Fruticultura 33: 12941299.

NEERAJA G; REDDY KM; REDDY MS; RAO VP. 2001. Influence of irrigation and nitrogen levels on bulb yield, nutrient uptake and nitrogen use efficiencies in rabi onion (Allium cepa). Indian Journal Agricultural Sciences 7: 109-11.

PORTO DRQ; CECILIO FILHO AB; MAY A; VARGAS PF. 2007. Acúmulo de macronutrientes pela cultivar de cebola "Superex" estabelecida por semeadura direta. Ciência Rural 37: 949-955.

PROCHNOW LI. 2008. Otimização do uso de nutrientes em solos de baixa fertilidade da região tropical. Informações Agronômicas 23: 1-7.

RAIJ B. 1991. Fertilidade do solo e adubação. Campinas: Ceres. 343p.

RESENDE GM; COSTA ND; PINTO JM. 2008. Produtividade e qualidade pós-colheita de cebola adubada com doses crescentes de nitrogênio e potássio. Horticultura Brasileira 26: 388-392.

SANTOS HG; JACOMINE PKT; ANJOS LHC; OLIVEIRA VA; OLIVEIRA JB; COELHO MR; LUMBRERAS JF; CUNHA TJF (ed). 2006. Sistema brasileiro de classificação de solos. 2. ed. Rio de Janeiro: Embrapa Solos, $306 \mathrm{p}$.

SEAGRI - Secretaria de Agricultura, Irrigação e Reforma Agrária do Estado da Bahia. 2013. Cotação Agrícola. Disponível em <http:// www.seagri.ba.gov.br/cotacao.asp $>$ Acesso em: 27 mai. 2013.

SINGH J, DHANKHAR BS. 1991. Effect of nitrogen, potash and zinc on storage loss of onion bulbs (Allium cepa). Vegetable Science 18: 16-23.

SINGH S, YADAV PK; SINGH B. 2004. Effect of nitrogen and potassium on growth and yield of onion (Allium cepa) cv. Pusa Red. Haryana Journal Horticultural Sciences 33: 308-309.

VIDIGAL SM. 2000. Adubação nitrogenada de cebola irrigada cultivada no verão - Projeto Jaiba, Norte de Minas Gerais. Viçosa: UFV. 136p (Tese doutorado).

YADAV GL; SHARMA PK; KUMAR S. 2003. Response of kharif onion to nitrogen and potash fertilization. News Letter National Horticultural Foundation 23: 4-6.

ZECA N; NASTO T; BALILU A. 2009. The influence of $\mathrm{N}$ sources and fertilization doses on dry onion (Allium cepa) yield and bulbs shelf life. Acta Horticulturae 830: 493-495. 